Nanoscale observation of delayering in alkane films

This article has been downloaded from IOPscience. Please scroll down to see the full text article.

2007 EPL 7926003

(http://iopscience.iop.org/0295-5075/79/2/26003)

View the table of contents for this issue, or go to the journal homepage for more

Download details:

IP Address: 128.206.162.204

The article was downloaded on 08/10/2010 at $16: 15$

Please note that terms and conditions apply. 


\title{
Nanoscale observation of delayering in alkane films
}

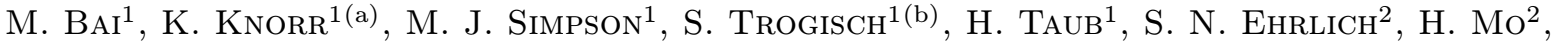 \\ U. G. VOLKMANn ${ }^{3}$ and F. Y. HANSEN ${ }^{4}$ \\ ${ }^{1}$ Department of Physics and Astronomy and University of Missouri Research Reactor, University of \\ Missouri-Columbia - Columbia, MO 65211, USA \\ ${ }^{2}$ National Synchrotron Light Source, Brookhaven National Laboratory - Upton, NY 11973 \\ ${ }^{3}$ Facultad de Física, Pontificia Universidad Católica de Chile - Santiago 22, Chile \\ ${ }^{4}$ Department of Chemistry, Technical University of Denmark - IK 207 DTU, DK-2800 Lyngby, Denmark
}

received 11 January 2007; accepted in final form 4 June 2007

published online 3 July 2007

PACS 68.08.Bc - Wetting

PACS 68.43.Hn - Structure of assemblies of adsorbates (two- and three-dimensional clustering)

PACS 87.64.Dz - Scanning tunneling and atomic force microscopy

\begin{abstract}
Tapping-mode Atomic Force Microscopy and synchrotron X-ray scattering measurements on dotriacontane $\left(n-\mathrm{C}_{32} \mathrm{H}_{66}\right.$ or C32) films adsorbed on $\mathrm{SiO}_{2}$-coated $\mathrm{Si}(100)$ wafers reveal a narrow temperature range near the bulk C32 melting point $T_{\mathrm{b}}$ in which a monolayer phase of C32 molecules oriented perpendicular to surface is stable. This monolayer phase undergoes a delayering transition to a three-dimensional (3D) fluid phase on heating to just above $T_{\mathrm{b}}$ and to a solid $3 \mathrm{D}$ phase on cooling below $T_{\mathrm{b}}$. An equilibrium phase diagram provides a useful framework for interpreting the unusual spreading and receding of the monolayer observed in transitions to and from the respective $3 \mathrm{D}$ phases.
\end{abstract}

Copyright (C) EPLA, 2007

Although a macroscopic theory of the wetting of solid surfaces by liquids has been developed some time ago [1], the general question of how molecular rotational and conformational degrees of freedom may influence the wetting of a liquid film interacting with a solid surface via van der Waals forces has not been investigated extensively and is poorly understood. Normal alkane molecules $\left[\mathrm{C}_{n} \mathrm{H}_{2 n+2}\right]$ of intermediate length $(15<n<50)$ provide model systems with which to address this issue. Torsional motion about $\mathrm{C}-\mathrm{C}$ bonds in the carbon backbone of the molecules results in conformational changes that play an essential role in determining the structure and melting of alkane monolayers [2]. Furthermore, the effect of these molecular conformational changes on wetting can be investigated systematically as a function of the alkane chain length $n$. As alkanes are the principal constituents of commercial lubricants $[3,4]$, their wetting properties are also of interest for lubricating nanoscale devices such as computer hard drives and micro-electro-mechanical systems.

It is generally believed that films of shorter alkanes $(n \leqslant 5)$ completely wet a solid substrate, i.e., form an

\footnotetext{
(a) Permanent address: Technische Physik, Universität des Saarlandes - D-66041 Saarbrücken, Germany.

(b) Permanent address: Qimonda Dresden GmbH \& Co. OHG Koenigsbruecker Strasse 180, D-01199 Dresden, Germany.
}

infinitely thick film, at the bulk triple point. This has only recently been demonstrated explicitly for the case of pentane $\left(n-\mathrm{C}_{5} \mathrm{H}_{12}\right)$ adsorbed on basal-plane graphite surfaces [5]. Experiments with intermediate-length alkanes $(15<n<50)$ adsorbed on $\mathrm{SiO}_{2}$ surfaces have not found triple-point wetting. Instead, liquid droplets have been observed by optical reflection microscopy at the bulk melting point [6-8]. Complete wetting of intermediate-length alkane films a few degrees above the bulk melting point was inferred indirectly by X-ray specular reflectivity $[6,9]$ and stray light intensity measurements [10].

In this report, we present direct evidence from tappingmode Atomic Force Microscopy (AFM) images that a film of an intermediate-length alkane, dotriacontane $\left(n-\mathrm{C}_{32} \mathrm{H}_{66}\right.$ or C32), does not completely wet a $\mathrm{SiO}_{2}$ surface at any temperature. Instead, we find a narrow temperature range near the C32 bulk melting point $T_{\mathrm{b}}$ where a monolayer phase in which the molecules are oriented perpendicular to the surface is stable. On heating just above $T_{\mathrm{b}}$, this monolayer phase undergoes a delayering transition to three-dimensional (3D) droplets that remain present up to their evaporation point. Moreover, the system shows an unusual re-entrant drying behavior in that the monolayer phase also undergoes a delayering transition to a 3D solid phase on cooling below $T_{\mathrm{b}}$. 


\section{(a) as dip-coated}

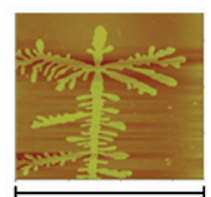

$20 \mu \mathrm{m}$

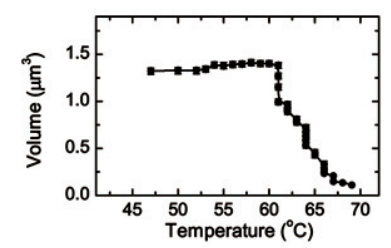

(b) $2^{\text {nd }}$ heating

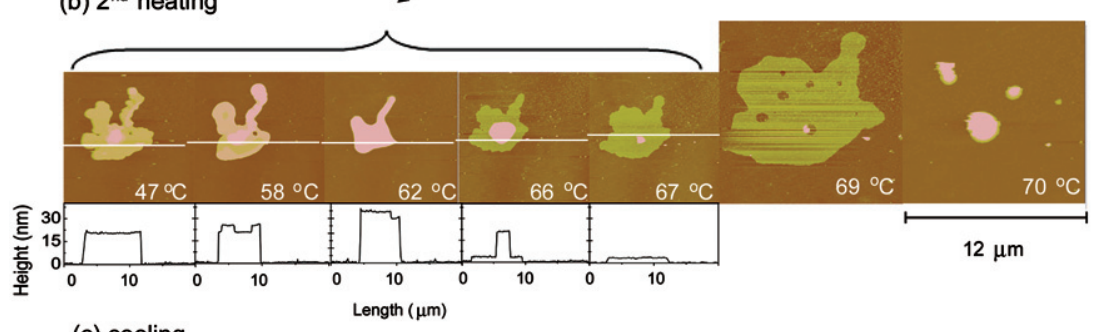

(c) cooling

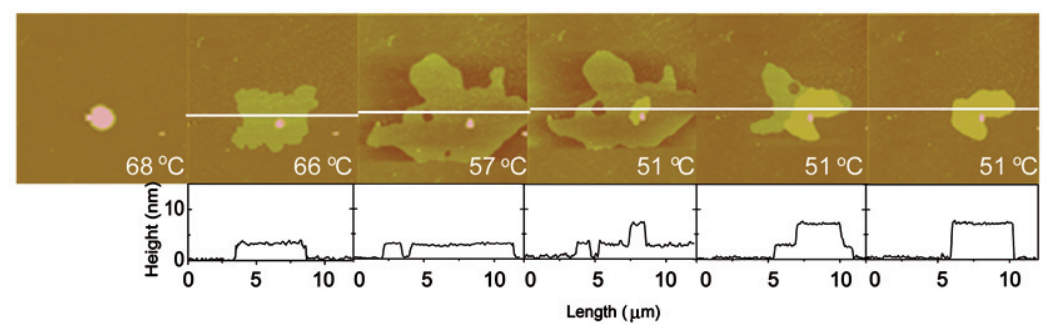

Fig. 1: Topographic AFM images of a low-coverage C32 film taken in the tapping mode. (a) At room temperature after deposition from solution; (b) temperature dependence of the images in the second heating cycle; and (c) images taken on cooling the sample after its second heating in (b). Height cross-sections shown below selected images were taken along the line indicated in white. The inset shows the temperature dependence of the particle volume in (b). All heights are measured relative to the one to two layers of $\mathrm{C} 32$ molecules that lie with their long-axis parallel and immediately adjacent to the $\mathrm{SiO}_{2}$ surface.

The AFM measurements on C32 films were performed with a Nanoscope IIIa (Veeco Instruments, Inc.) operating in the tapping mode. Using silicon cantilevers with a resonance frequency typically of $\sim 33 \mathrm{kHz}$, we simultaneously recorded AFM images of topography and phase angle (cantilever oscillation relative to the drive) as a function of temperature. The samples reported on here were made by dip-coating an acid-cleaned, electronicgrade $\mathrm{Si}(100)$ substrate in a solution of $\mathrm{C} 32$ dissolved in heptane $(\mathrm{C} 7)[11,12]$ with alkane purity $>99.9 \%$. The $\mathrm{Si}(100)$ wafers typically had native oxide coatings with thickness in the range 12-25 $\AA$ [11]. The samples were mounted in a closed cell under a dry nitrogen atmosphere so that the total amount of alkane was kept constant during the AFM measurements, although it was impossible to measure the C32 vapor pressure.

In fig. 1(a), we see a topographic AFM image of a sample at room temperature taken after dip coating and the subsequent evaporation of the $\mathrm{C} 7$ solvent. The image shows an island of adsorbed C32 having a "dragonfly" shape. Topographic cross-sections indicate the height of this feature to be $\sim 4.2 \mathrm{~nm}$ after calibration using the AFM contact mode $[12,13]$ and X-ray specular reflectivity measurements [11]. Because this height is approximately equal to the all-trans length of the C32 molecule, we interpret the dragonfly feature as consisting of a single layer of molecules oriented with their long axis perpendicular to the surface. Hereafter, we refer to such a structure as a "perpendicular monolayer." This feature is qualitatively similar to the fractal-like islands previously observed by AFM at low coverages of $\mathrm{C} 30$ on $\mathrm{SiO}_{2}$ [14].

$\mathrm{X}$-ray specular reflectivity [11] and contact mode AFM measurements [12] at room temperature indicate that these perpendicular monolayer islands reside on one to two layers of $\mathrm{C} 32$ molecules oriented with their long axis parallel to the $\mathrm{SiO}_{2}$ surface. Because of their larger area of contact, the parallel molecules are presumably bound more strongly by van der Waals forces to the $\mathrm{SiO}_{2}$ surface than the perpendicular molecules are to the parallel layers. Our evidence suggests that at room temperature the parallel molecules form layers covering virtually the entire $\mathrm{SiO}_{2}$ surface in contrast to the partial layers of perpendicular molecules. In the tapping mode, we did not observe any holes in the parallel layers that allowed determination of their thickness.

After heating this sample slowly $(1 \mathrm{~K} / \mathrm{min})$ above the bulk melting point of $\mathrm{C} 32$ at $68^{\circ} \mathrm{C}$ and returning at the same rate to $47^{\circ} \mathrm{C}$, we find a qualitative change in topography. As shown in the first image of fig. 1(b), a typical scan area no longer contains perpendicular monolayer islands but only mesa-shaped particles that crosssections indicate have a height of $20-40 \mathrm{~nm}$. Previous 
X-ray diffraction [11] and contact mode AFM [12] measurements indicate that these particles have an orthorhombic structure in which the $\mathrm{C} 32$ molecules are aligned perpendicular to the $\mathrm{SiO}_{2}$ surface. Because these mesa-shaped orthorhombic particles appear on annealing and retain their structure on cooling to room temperature as well as in a subsequent heating cycle, we believe they represent the equilibrium structure at room temperature. We also note that in true equilibrium the solid mesa-shaped particles would be faceted rather than having an irregular shaped boundary as in fig. 1(b). However, the X-ray diffraction scans discussed below offer evidence that on heating the majority of the material within the particles is in thermal equilibrium.

Figure 1(b) follows the evolution of one of the 3D particles in a second slow heating of the film (the temperature is ramped upward at a rate always below $0.1 \mathrm{~K} / \mathrm{min}$ between AFM images and the recording of each image lasts $\sim 4.5$ min during which the temperature is kept constant). Below the first five AFM images, we show a cross-section of the film height along the white line drawn in the image. The cross-section shows that the particle has a flat top of various heights indicating a solid particle. As the sample is heated from $47^{\circ} \mathrm{C}$ to $58^{\circ} \mathrm{C}$, the particle boundary becomes smoother, consistent with the material on the particle sides coming closer to equilibrium. At $\sim 61^{\circ} \mathrm{C}$, a dramatic change in the particle occurs as indicated by an abrupt decrease in its volume (see inset). Also, as shown in the cross-section below the image at $62{ }^{\circ} \mathrm{C}$, there is a small increase in its height along the scan line as well as a continued smoothing of its boundary. Apparently, the material lost from the solid particle enters a 3D gas phase or an interfacial (2D) gas phase in which it is invisible to AFM.

Between $64{ }^{\circ} \mathrm{C}$ (not shown) and $66^{\circ} \mathrm{C}$ in fig. 1(b), we observe a new phenomenon, not seen at lower temperatures, in which material begins to spread out of the flat-topped particle at the expense of its volume. The cross-section at $66^{\circ} \mathrm{C}$ indicates that the spreading layer has a height of $\sim 4.2 \mathrm{~nm}$, which we again interpret as being a perpendicular monolayer and the remaining particle, now with an even smoother boundary, has a height of four perpendicular layers. The perpendicular monolayer continues to spread on heating until, at $\sim 69^{\circ} \mathrm{C}$, it reaches its maximum lateral extent and the material in the mesashaped particle has been nearly exhausted. This behavior contrasts with that reported in ref. [7] where a perpendicular monolayer of an intermediate-length alkane (C30) spread outward from a fluid droplet with a spherically shaped cap on cooling just below the bulk melting point.

At $\sim 69^{\circ} \mathrm{C}$, holes develop in the perpendicular monolayer; and, at $70^{\circ} \mathrm{C}$, there is an abrupt transition to $3 \mathrm{D}$ droplets identified both by their spherical profile ${ }^{1}$ and circular perimeter as well as by a large positive increase in the phase angle measured. In other similarly prepared

\footnotetext{
${ }^{1}$ Cross-sections are not shown for the hemispherically shaped $3 \mathrm{D}$ droplets at $70^{\circ} \mathrm{C}$ (heating) and $68^{\circ} \mathrm{C}$ (cooling) because of the large amount of noise due to tip capture by the fluid.
}

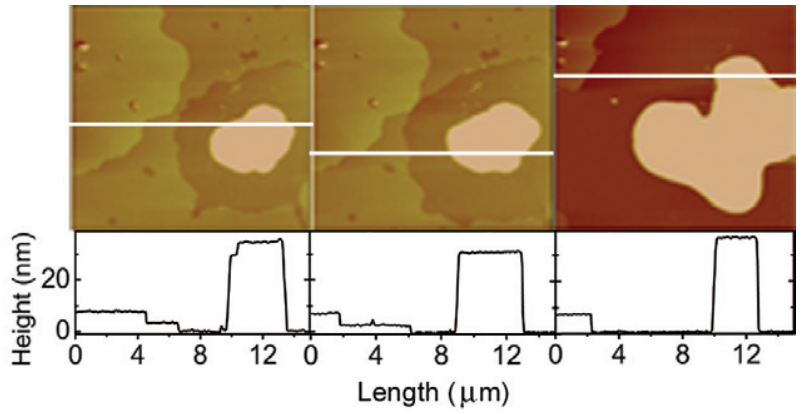

Fig. 2: Topographic AFM images of a low-coverage C32 film taken in the noncontact mode at $55^{\circ} \mathrm{C}$ during the cooling half of the first heating/cooling cycle. The first two images took about 4.5 minutes, and the last image was recorded after waiting two hours without scanning. Height cross-sections shown below the images were taken along the white lines. These images provide evidence that the mesa-shaped bulk particle on the right grows in size at the expense of the regions of one and two layers of perpendicular molecules on the left by molecular transport through an interfacial gas phase.

samples that we investigated to higher temperatures, we observed no wetting of C32 to occur up to a temperature of $85^{\circ} \mathrm{C}$ at which there is significant thermal desorption into the $3 \mathrm{D}$ gas phase [10].

On cooling this sample just below the bulk melting point at $68^{\circ} \mathrm{C}$, we see in fig. 1 (c) that a perpendicular monolayer begins to spread outward from one of the droplets as the droplet volume decreases, a behavior similar to that reported in ref. [7] (see above). Now the source of the spreading monolayer is a hemispherical droplet $^{1}$ rather than a flat-topped particle. Interestingly, we have been able to follow this spreading behavior to lower temperatures than in ref. [7]. At $57^{\circ} \mathrm{C}$, the material in the droplet is nearly exhausted and holes begin to appear in the monolayer. With further cooling of the sample to $54^{\circ} \mathrm{C}$, we observe a second perpendicular layer form on top of the first. At this point, the combined volume of the two layers begins to decrease due to loss of material to the interfacial gas phase, and the film kinetics slows. The last three AFM images at $51{ }^{\circ} \mathrm{C}$ show the subsequent evolution as a function of time. The second perpendicular layer grows in area at the expense of the first layer as their combined volume continues to decrease until the two layers match boundaries to form a bilayer island.

This loss of material on cooling of the bilayer in fig. 1(c) may be due to its having a sub critical volume necessary to nucleate a solid 3D particle, while 3D particles are formed around nuclei with a super critical volume outside the scan area. As depicted in fig. 2 for another low-coverage sample on cooling, we observe growth of thicker mesashaped particles whose volume increases over a period of hours. A possible mechanism for this growth is transport of C32 from monolayer islands of perpendicular molecules through an interfacial gas phase. 


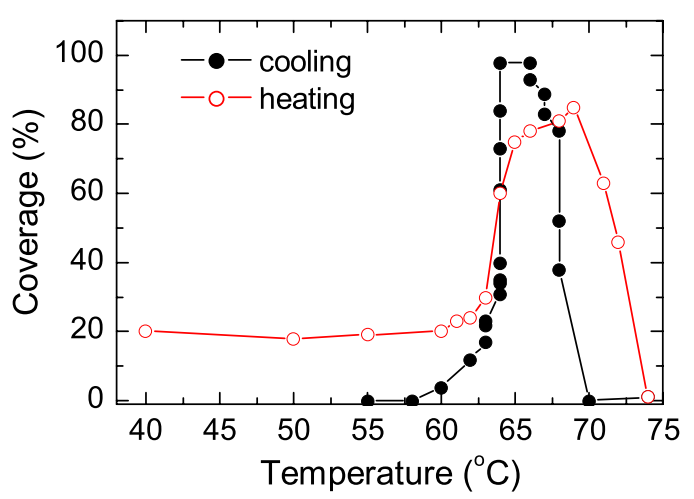

Fig. 3: Plot of the fraction of the scan area occupied by a layer of perpendicular molecules for a high-coverage C32 film during its first heating/cooling cycle.

We interpret the sequence of AFM images in fig. 1(b) as indicating the stability of a monolayer phase of C32 molecules oriented perpendicular to the surface in the temperature range from $64{ }^{\circ} \mathrm{C}$ to $69^{\circ} \mathrm{C}$. On heating, the kinetics of the transition from the $3 \mathrm{D}$ mesa-shaped particles to the monolayer phase is sufficiently slow that we are able to view the growth of the monolayer phase in time. Similarly, on cooling, the transition from 3D liquid droplets to the perpendicular monolayer phase shown in fig. 1(c) is kinetically hindered so that we again view the growth of the monolayer as an outward spreading from a $3 \mathrm{D}$ droplet that has a higher chemical potential.

We have also performed similar heating/cooling cycles on higher-coverage samples. For these, we observe spreading of a perpendicular monolayer phase to begin on heating above $\sim 62{ }^{\circ} \mathrm{C}$; however, the number and size of the source particles are now sufficient to allow the perpendicular monolayer phase to fill a larger fraction of the scan area. The delayering transition to $3 \mathrm{D}$ droplets is again initiated by the development of holes in the perpendicular monolayer followed by a decrease in its area. This behavior is similar to the recession of a perpendicular monolayer as previously observed for a C30 film heated above its bulk melting point [7].

In fig. 3, we have plotted the fraction of the scan area occupied by the perpendicular monolayer structure as a function of temperature on heating and cooling one of these higher coverage samples. It is evident that there is a well-defined temperature range, $64^{\circ} \mathrm{C}$ to $69^{\circ} \mathrm{C}$, in which the perpendicular monolayer structure occupies over $80 \%$ of the scan area $\left(12.5 \times 12.5 \mu \mathrm{m}^{2}\right)$. The approach to equilibrium is slow so that on heating we observe the spreading of the monolayer at the onset of the transition to the perpendicular monolayer phase and then recession of the monolayer in anticipation of the transition to the 3D fluid phase. On cooling, these kinetic processes are reversed so that, on approaching the transition to the monolayer phase from above, the monolayer spreads with some hysteresis and then at lower temperature recedes in anticipation of the transition to the 3D solid phase. These results are consistent with those described above for a lower coverage sample. They support our interpretation of a narrow range in temperature just below the bulk C32 melting point in which a monolayer phase of C32 molecules oriented perpendicular to the surface is stable.

We have also investigated the structural changes in the C32 films observed by AFM using grazing-incident-angle synchrotron X-ray diffraction. Due to the much larger area of the film sampled by the incident X-ray beam compared to that of an AFM image $\left(\sim 10^{5}\right.$ times larger $)$, the X-ray patterns give an average structure that can be helpful in determining the equilibrium phases of the film. Measurements were conducted at beam line 6ID-B of the Advanced Photon Source using an X-ray wavelength of $0.765 \AA$ and a two-dimensional detector. The incident beam made an angle of $\sim 0.1^{\circ}$ with the $\mathrm{SiO}_{2}$ surface, which is slightly greater than or comparable to the critical angle for $\mathrm{C} 32\left(0.077^{\circ}\right)$ and $\mathrm{SiO}_{2}\left(0.11^{\circ}\right)$, respectively. The sample had about the same coverage and was prepared similarly to the one used in the AFM measurements of fig. 1(b), including the initial heating/cooling cycle.

In fig. 4(a), we show scans of the X-ray intensity taken with the wave vector transfer $\mathbf{Q}$ aligned parallel to the $\mathrm{SiO}_{2}$ surface. At each value of $Q$, the intensity has been integrated over a narrow range $0.02 \AA^{-1}$ to $0.04 \AA^{-1}$ in a direction normal to the surface in order to improve statistics but to exclude contributions from dynamical scattering near the C32 critical angle. Below a temperature of $56^{\circ} \mathrm{C}$, we see a diffraction pattern characteristic of a polycrystalline film consisting of three features: a dominant peak at $Q \sim 1.51 \AA^{-1}$ (labeled peak 2), a shoulder on its leading edge caused by a peak at $\sim 1.49 \AA^{-1}$ (labeled peak 1), and a broader peak at $Q \sim 1.66 \AA^{-1}$. These features cannot be indexed by the bulk monoclinic structure reported for C32 at room temperature [15].

The decomposition of the X-ray scans parallel to the surface into peaks 1 and 2 is illustrated by the dashed curves plotted underneath the scan at $47^{\circ} \mathrm{C}$ in fig. 4(a). In fig. 4(b), we have plotted the position of these two peaks as a function of temperature. We see that the position of the dominant peak 2 evolves smoothly to a value of $Q=1.49 \AA^{-1}$ upon heating to $68^{\circ} \mathrm{C}$ where fig. 4(a) shows a weak and broad peak. As this is the last diffraction feature to remain before the delayering transition to a $3 \mathrm{D}$ droplet as observed in the AFM images of fig. 1(b), we tentatively identify the weak and broad peak at $68^{\circ} \mathrm{C}$ with the equilibrium perpendicular monolayer phase. Its position is close to the value of $1.51 \AA^{-1}$ found for the perpendicular monolayer phase that has hexagonal symmetry in the surface freezing effect of bulk C32 [16].

We have found that the position and intensity of the shoulder observed below $57^{\circ} \mathrm{C}$ at $Q=1.50 \AA^{-1}$ depends somewhat on the part of the C32 film illuminated by the $\mathrm{X}$-ray beam. We speculate that it could represent a remnant of the metastable dragonfly phase in fig. 1(a) that, due to "pinning" by defects, did not participate in the delayering transition that occurred on heating during the 

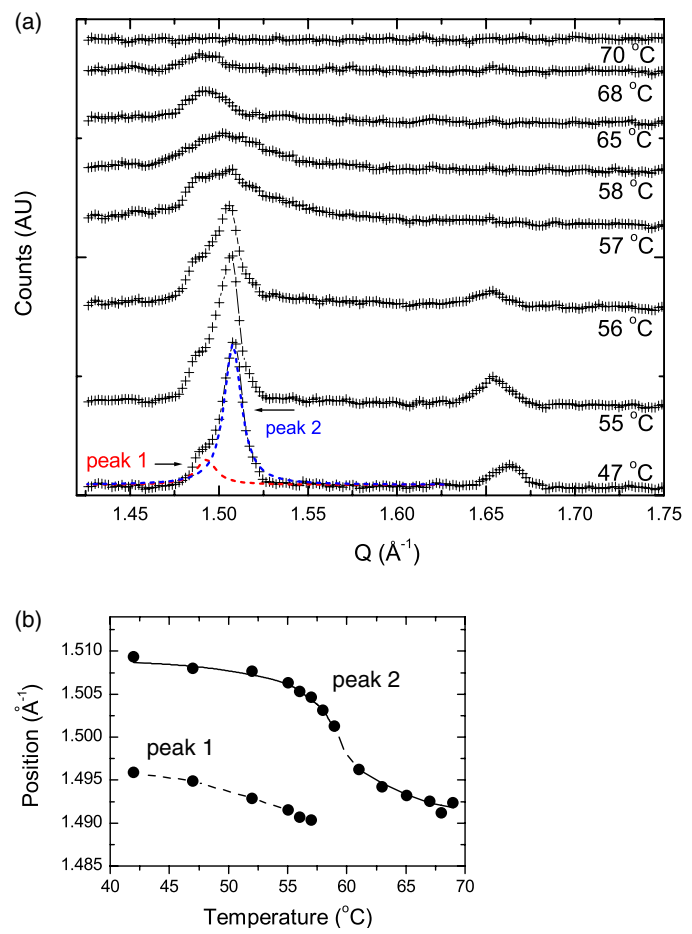

Fig. 4: (a) Temperature dependence of the in-plane synchrotron X-ray diffraction scans of a C32 film prepared similarly to that in fig. 1. Intensities are calculated from the $2 \mathrm{D}$ detector after integrating over a limited $Q$ range in a direction perpendicular to the $\mathrm{SiO}_{2}$ surface as described in the text. Dashed lines indicate the decomposition of the main diffraction peak into a shoulder (peak 1) and the dominant peak 2. (b) Plot of the position in $\AA^{-1}$ of peaks 1 and 2 vs. temperature. Solid and dashed curves are guides to the eye. The peak 2 data represent the orthorhombic phase $C^{\prime}$ up to $\sim 57^{\circ} \mathrm{C}$ at which point the transition to the rotator $R^{\prime}$ phase occurs. The points above $60{ }^{\circ} \mathrm{C}$ correspond to the equilibrium perpendicular monolayer phase. Peak 1 represents the metastable perpendicular monolayer phase. It is not clear what happens to this metastable monolayer phase at higher temperature.

first thermal cycle. Alternatively, it might result from a metastable perpendicular monolayer phase that, due to slow kinetics or pinning, did not delayer on cooling during the first cycle. We note that the position of the weak and broad peak at $68^{\circ} \mathrm{C}$ that we have identified with the equilibrium perpendicular monolayer phase is close to that of the putative metastable monolayer. It is not clear what happens to this metastable monolayer phase at higher temperature.

Again, using the AFM images as a guide, we identify the two stronger peaks in the X-ray scans of fig. 4(a) below a temperature of $57^{\circ} \mathrm{C}$ with a crystalline phase $C^{\prime}$ corresponding to an ensemble of $3 \mathrm{D}$ mesa-shaped particles similar to the one in fig. 1(b) and having a distribution of heights. Indexing the lower $\left(Q=1.51 \AA^{-1}\right)$ and upper $\left(Q=1.66 \AA^{-1}\right)$ of these peaks as the (110) and (200) reflections of an orthorhombic unit cell, respectively, we obtain lattice constants $a=7.57 \AA$ and $b=4.98 \AA$ in reasonable agreement with those found for orthorhombic particles of C32 growing on a $\mathrm{Ag}(111)$ surface [17].

We have tried to analyze the X-ray intensity along scans (not shown) directed nearly perpendicular to the $\mathrm{SiO}_{2}$ surface at the position of peak 1 and the dominant peak 2 in fig. 4(a) as well as at $Q=0$. These scans have proved difficult to model quantitatively due to the vertical disorder in the film and the height dispersion of the 3D particles present. Nevertheless, at $55^{\circ} \mathrm{C}$, the perpendicular scans clearly show that the intensity of peaks 1 and 2 falls monotonically to zero over a range $\Delta Q_{z} \approx 0.14 \AA^{-1}$, corresponding to a film thickness $2 \pi / \Delta Q_{z} \approx 45 \AA$, which is on the order of the all-trans length of the C32 molecule. This behavior is consistent with both peaks being contributed by regions of the film in which the molecules are oriented perpendicular to the $\mathrm{SiO}_{2}$ surface rather than from the one to two layers of parallel molecules underneath.

As shown in fig. $4(\mathrm{a})$, between $56^{\circ} \mathrm{C}$ and $58^{\circ} \mathrm{C}$, we observe a transition from the double-peak feature near $Q \sim 1.5 \AA^{-1}$ to a single broad peak and the disappearance of the weaker peak at $Q \sim 1.66 \AA^{-1}$. These changes indicate a transition in the film from the orthorhombic structure described above to a phase characterized by a higher symmetry (probably a hexagonal structure) and much a shorter coherence length than in the lowtemperature $C^{\prime}$ phase. Following previous suggestions of a rotator phase for interfacial alkane molecules of intermediate length $[9,10]$, we interpret the changes in the X-ray scans in the range $56-58^{\circ} \mathrm{C}$ as indicating a transition from the $3 \mathrm{D}$ crystalline phase $C^{\prime}$ to a $3 \mathrm{D}$ rotator (plastic) phase $R^{\prime}$ phase in which the C32 molecules are orientationally disordered about their long axis. As discussed above, the $R^{\prime}$ subsequently undergoes a transition to the perpendicular monolayer phase on heating to $\sim 64^{\circ} \mathrm{C}$ as indicated by the AFM images.

We point out that the transition from the interfacial orthorhombic crystalline phase $C^{\prime}$ to the rotator phase $R^{\prime}$ phase occurs about $7^{\circ} \mathrm{C}$ below that of the monoclinicto-triclinic crystalline-to-rotator phase transition in bulk $\mathrm{C} 32$ at $64^{\circ} \mathrm{C}$ [18]. The $R^{\prime}$ phase likely has a different structure and a shorter coherence length than the bulk triclinic rotator phase. The flat tops of the particles in the AFM images of fig. 1(b) in the temperature range $58-66^{\circ} \mathrm{C}$ suggest that the $R^{\prime}$ phase has a layered structure like the orthorhombic $C^{\prime}$ phase, while the broad diffraction peak at $58^{\circ} \mathrm{C}$ implies considerably more translational disorder within a layer than in the $C^{\prime}$ phase. Furthermore, the abrupt change in particle volume in this temperature range (see inset to fig. 1(b)) indicates a rapid exchange of molecules between the $R^{\prime}$ and gas phases as well as a high mobility of the molecules within the $R^{\prime}$ phase. This high mobility is consistent with the smoothing of the particle boundary observed in the AFM images of fig. 1(b) in the temperature range $58-66^{\circ} \mathrm{C}$.

It is useful to summarize our results in a model equilibrium phase diagram shown in fig. 5 where we have plotted the $\mathrm{C} 32$ chemical potential $\mu$ measured with respect to 


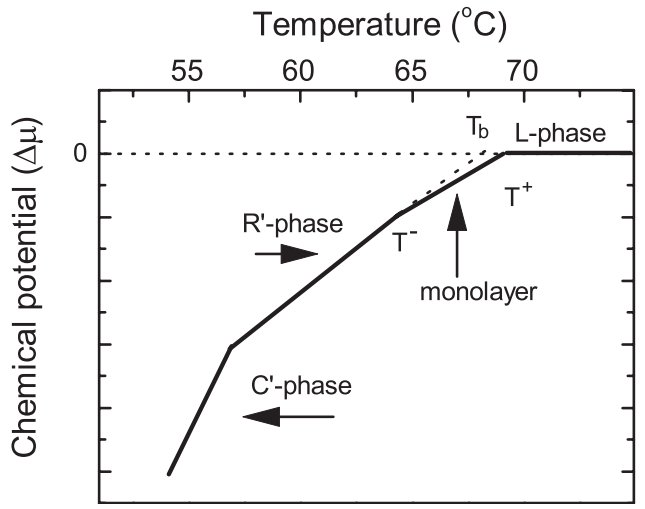

Fig. 5: Proposed phase diagram for the submonolayer C32 film plotted in the $\mu-T$ plane where the chemical potential $\mu$ is measured relative to that of the bulk C32 liquid $(L) . C^{\prime}$ and $R^{\prime}$ refer to the interfacial bulk C32 crystalline and rotator phases, respectively. The solid line segment between $T^{-}$and $T^{+}$denotes the stability region of the perpendicular monolayer phase.

that of the bulk liquid as a function of temperature. In constructing this diagram, we assume that the underlying one to two layers of parallel molecules immediately adjacent to the $\mathrm{SiO}_{2}$ surface remain in a solid state and that there is a coexisting interfacial (or 2D) gas phase throughout the temperature range considered. The solid black line segments labeled $C^{\prime}, R^{\prime}$, monolayer, and $L$ therefore represent the coexistence lines of the bulk crystalline, rotator, perpendicular monolayer, and liquid phases, respectively, with the interfacial vapor phase (see fig. 7 in ref. [5]). The prime indicates interfacial structures distinct from the freestanding bulk phases. We suggest that there is a firstorder transition from the $3 \mathrm{D} R^{\prime}$ phase to a perpendicular monolayer phase at a temperature $T^{-} \sim 64{ }^{\circ} \mathrm{C}$ and from this phase to the $3 \mathrm{D} L$ phase at $T^{+} \sim 69^{\circ} \mathrm{C}$. The solid line segment between $T^{-}$and $T^{+}$represents the stability region of the perpendicular monolayer phase. We interpret the monolayer spreading behavior observed as the transition temperatures $T^{-}$and $T^{+}$are crossed in the direction toward the perpendicular monolayer phase to result from a slow relaxation to equilibrium. Similarly, we attribute the recession of the monolayer to a slow relaxation to the respective $3 \mathrm{D}$ phases as these phase boundaries are crossed in the opposite direction. Both the $R^{\prime}$ and perpendicular monolayer phases are characterized by shorter-range translational order than in the $C^{\prime}$ phase; but, as indicated by the slope of their co-existence lines in the phase diagram, they have lower entropy than the nonwetting bulk liquid phase.

Preliminary AFM and synchrotron X-ray measurements indicate that a similar phase diagram applies to submonolayer C32 deposited from solution onto highly oriented pyrolytic graphite as well as to $\mathrm{C} 24, \mathrm{C} 30$, and C36 deposited on the same $\mathrm{SiO}_{2}$ surfaces that we have used in this study. These results raise a number of interesting questions: Why does the bulk liquid fail to wet the underlying parallel layers of molecules immediately adjacent to the $\mathrm{SiO}_{2}$ surface and is the delayering transition of the perpendicular monolayer phase related to conformational changes in the molecules? What are the microscopic mechanisms driving the spreading and receding of the perpendicular monolayer phases? And at what (shorter) alkane chain length will a crossover to complete wetting occur as observed for pentane on graphite?

$$
* * *
$$

We thank D. S. RoBinson for technical support at the 6-ID beam line of the Advanced Photon Source. This work was supported by the U.S. National Science Foundation under Grant No. DMR-0411748, by the Chilean government under FONDECYT Grant Nos. 1010548 and 701058, and by the U.S. Department of Energy under Contract Nos. W-7405-Eng-82 and W-31-109-Eng-38.

\section{REFERENCES}

[1] De Gennes P. G., Rev. Mod. Phys., 57 (1985) 827.

[2] Hansen F. Y., Herwig K. W., Matthies B. and Taub H., Phys. Rev. Lett., 83 (1999) 2362.

[3] Landman Uzi, Luedtke W. D., Ouyang J. and Xia T. K., Jpn. J. Appl. Phys., 32 (1993) 1444.

[4] Mondello M. and Grest G. S., J. Chem. Phys., 103 (1995) 7156.

[5] Kruchten F., Knorr K., Volkmann U. G., Taub H., Hansen F. Y., Matthies B. and Herwig K. W., Langmuir, 21 (2005) 7507.

[6] Merkl C., Pfohl T. and Riegler H., Phys. Rev. Lett., 79 (1997) 4625.

[7] Lazar P., Schollmeyer H. and Riegler H., Phys. Rev. Lett., 94 (2005) 116101.

[8] Lazar P. and Riegler H., Phys. Rev. Lett., 95 (2005) 136103.

[9] Schollmeyer H., Struth B. and Riegler H., Langmuir, 19 (2003) 5042.

[10] Volkmann U. G., Pino M., Altamirano L. A., Taub H. and Hansen F. Y., J. Chem. Phys., 116 (2002) 2107.

[11] Mo H., Taub H., Volkmann U. G., Pino M., Ehrlich S. N., Hansen F. Y., Lu E. and Miceli P., Chem. Phys. Lett., 377 (2003) 99.

[12] Trogisch S., Simpson M. J., Taub H., Volkmann U. G., Pino M. and Hansen F. Y., J. Chem. Phys., 123 (2005) 154703.

[13] Bai M., Trogisch S., Magonov S. and Taub H., in preparation.

[14] Holzwarth A., Leporatti S. and Riegler H., Europhys. Lett., 52 (2000) 643.

[15] Craig S. R., Hastle G. P., Roberts K. J. and Sherwood J. N., J. Mater. Chem., 4 (1994) 977.

[16] Ocko B. M., Wu X. Z., Sirota E. B., Sinha S. K., Gang O. and Deutsch M., Phys. Rev. E, 55 (1997) 3164.

[17] Mo H., Trogisch S., Taub H., Ehrlich S. N., Volmmann U. G., Hansen F. Y. and Pino M., Phys. Status Solidi (a), 201 (2004) 2375.

[18] Sirota E. B., King H. E. jr., Singer D. M. and Shao H. H., J. Chem. Phys., 98 (1993) 5809. 